\title{
Metallic-glass-matrix composite structures with benchmark mechanical performance
}

\author{
Joseph P. Schramm, ${ }^{1}$ Douglas C. Hofmann, ${ }^{1,2, a)}$ Marios D. Demetriou, ${ }^{1}$ and \\ William L. Johnson ${ }^{1}$ \\ ${ }^{1}$ Keck Laboratory, California Institute of Technology, Pasadena, California 91125, USA \\ ${ }^{2}$ Engineering and Science Directorate, Enterprise Engineering Division, Jet Propulsion Laboratory, \\ California Institute of Technology, Pasadena, California 91109, USA
}

(Received 19 October 2010; accepted 4 November 2010; published online 16 December 2010)

\begin{abstract}
Metallic-glass-matrix composites demonstrating unusual combination of high strength, high toughness, and excellent processability are utilized to fabricate cellular structures of egg-box topology. Under compressive loading, the egg-box panels are capable of undergoing extensive plastic collapse at very high plateau stresses enabling absorption of large amounts of mechanical energy. In terms of specific mechanical energy absorbed, the present panels far outperform panels of similar topology made of aluminum or fiber-reinforced polymer composites, and even surpass steel structures of highly buckling-resistant topologies, thus emerging among the highest performance structures of any kind. (C) 2010 American Institute of Physics. [doi:10.1063/1.3521412]
\end{abstract}

The design of strong, lightweight structures with the capacity to absorb mechanical energy has been the focus of structural engineers and materials scientists for over three decades. Such an endeavor demands optimization of both architectural design and material performance to achieve high-strength, low-density, and plastically deformable cellular structures. Specifically, buckling-resistant architectures are sought, made of materials with high specific strength and toughness capable of undergoing extensive plastic collapse under stress. Significant progress in the design of architectures highly resistant to buckling has been reported to date, ${ }^{1}$ but materials suitable for the fabrication of such structures are either insufficiently strong or insufficiently tough to enable high mechanical dissipation during collapse. This is because processability and strength, as well as toughness and strength, are usually mutually exclusive for essentially all classes of materials. ${ }^{2}$ Materials of choice for the development of such architectures are presently limited to ductile crystalline metals, and mostly to aluminum alloys and lowcarbon steels. ${ }^{1}$ Aluminum alloys exhibit superb processability and adequate toughness; mild steels exhibit superb toughness and sufficient processability; both however suffer from very low yield strengths $(<500 \mathrm{MPa}){ }^{2}$ Consequently, the mechanical energy absorption capacity of structures made of such materials is generally suboptimal. In this article, toughened metallic-glass-matrix composites (MGMCs) ${ }^{3}$ with excellent processability are utilized for the development of structurally advanced cellular cores.

A collapsing cellular structure attains the highest possible failure stress when the structural members (struts) deform by plastic yielding. ${ }^{4}$ As such, elastic buckling and brittle fracture of struts should be avoided upon collapse. Buckling can occur either as a consequence of high strut elasticity (high material elastic limit) or due to a weakly constrained architecture (limited nodal connectivity). For example, periodic architectures (e.g., honeycombs) are more strongly constrained and thus more buckling resistant than stochastic architectures (e.g., foams), while certain periodic

\footnotetext{
${ }^{\text {a) }}$ Author to whom correspondence should be addressed. Electronic mail: dch@jpl.nasa.gov.
}

configurations (e.g., trusses) are more buckling resistant than others (e.g., textiles). ${ }^{5}$ For a given architecture, the critical strut slenderness ratio (and associated relative density) below which the structure becomes unstable against buckling is determined by the material elastic limit. Specifically, for a periodic architecture, the critical slenderness ratio scales inversely with the elastic limit. ${ }^{6}$ Hence, the characteristic scale $E / \sigma_{y}$ ( $E$ is Young's modulus and $\sigma_{y}$ the yield strength) is employed here to characterize the buckling resistance of various materials. ${ }^{7}$ Brittle fracture, on the other hand, occurs when struts fail catastrophically by unstable crack propagation. Such failure occurs when the material lacks sufficient fracture toughness $\left(K_{I C}\right)$, or exhibits very high yield strength, so that cracks nucleated at the tension side of a bent strut cannot be plastically arrested. For a strut to resist such catastrophic failure, the plastic zone developing ahead of an opening crack tip should have dimensions comparable to the width of the strut. The plastic zone size, $K_{I C}{ }^{2} / \pi \sigma_{y}{ }^{2}{ }^{2}$ is employed here as the characteristic length scale to quantify the resistance of various materials against fracture. If struts avoid both buckling and fracture and yield plastically during collapse, the structure will exhibit the maximum possible plateau stress and absorb the maximum possible energy associated with its architecture and relative density. The material characteristic scale that quantifies the specific plateau

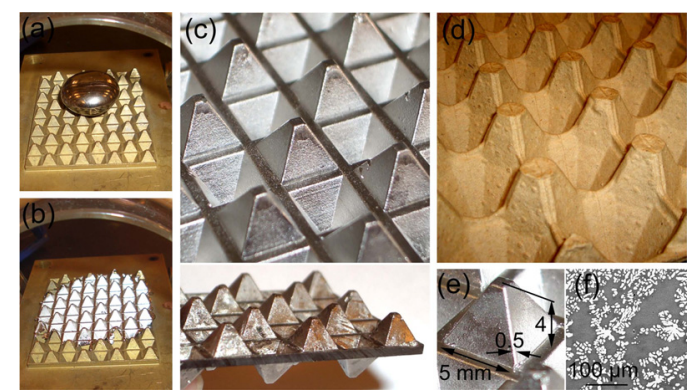

FIG. 1. (Color online) The semisolid-forging setup used to fabricate eggboxes. (a) and (b) Lower die tool shown before and after forging of a DH1 egg-box panel (c) and (d) Comparison of a DH1 egg-box panel of 0.12 relative density (c) against a cardboard egg-box carton (d). (e) Geometry of a DH1 egg-box cell (f) Scanning electron micrographs revealing the underlying microstructure of an as-forged DH1 egg-box cell section. 
TABLE I. Properties of various alloys. (Density $\rho$, Young's modulus $E$, yield strength $\sigma_{y}$, fracture toughness $K_{c}$, inverse of elastic strain limit $E / \sigma_{y}$, plastic zone size $K_{c}{ }^{2} / \pi \sigma_{y}{ }^{2}$, and specific strength $\sigma_{y} / \rho$.

\begin{tabular}{|c|c|c|c|c|c|c|c|}
\hline & $\begin{array}{c}\rho \\
\left(\mathrm{g} / \mathrm{cm}^{3}\right)\end{array}$ & $\begin{array}{c}E \\
(\mathrm{GPa})\end{array}$ & $\begin{array}{c}\sigma_{y} \\
(\mathrm{MPa})\end{array}$ & $\begin{array}{c}K_{c} \\
\left(\mathrm{MPa} \mathrm{m}^{1 / 2}\right)\end{array}$ & $\begin{array}{c}E / \sigma_{y} \\
(-)\end{array}$ & $\begin{array}{c}K_{c}^{2} / \pi \sigma_{y}^{2} \\
\quad(\mathrm{~mm})\end{array}$ & $\begin{array}{l}\sigma_{y} / \rho \\
(\mathrm{J} / \mathrm{g})\end{array}$ \\
\hline AISI 1020 & 7.9 & 200 & 350 & 140 & 571 & 50 & 44 \\
\hline Ti-6Al-4V & 4.4 & 114 & 1100 & 43 & 103 & 0.5 & 250 \\
\hline $\mathrm{Zr}_{41.2} \mathrm{Ti}_{13.8} \mathrm{Cu}_{12.5} \mathrm{Ni}_{10} \mathrm{Be}_{22.5}$ (Vitreloy-1) & 6.1 & 97 & 1737 & 55 & 56 & 0.3 & 285 \\
\hline $\mathrm{Zr}_{36.6} \mathrm{Ti}_{31.4} \mathrm{Nb}_{7} \mathrm{Cu}_{5.9} \mathrm{Be}_{19.1}(\mathrm{DH} 1)$ & 5.6 & 85 & 1512 & 150 & 56 & 3.1 & 270 \\
\hline
\end{tabular}

stress and specific energy absorption capacity (in $\mathrm{J} / \mathrm{g}$ ) of a plastically collapsing structure is the specific strength, $\sigma_{y} / \rho$, which is also adopted here for comparison.

These characteristic scales are estimated for mild steel AISI 1020, aluminum alloy Al 6061 T6, titanium alloy Ti6Al-4V, metallic glass (Vitreloy-1), and MGMC DH1, and are listed in Table I in order of increasing $\sigma_{y} / \rho$. As seen in the table, the increasing $\sigma_{y} / \rho$ is accompanied by a decreasing $E / \sigma_{y}$ and $K_{c}{ }^{2} / \pi \sigma_{y}{ }^{2}$. Although the decrease in $E / \sigma_{y}$ with increasing $\sigma_{y} / \rho$ appears significant, it may not be overly restrictive. For example, even for structures with limited nodal connectivity (e.g., triangular honeycombs), a low value of $E / \sigma_{y}=56$ for Vitreloy- 1 is associated with a critical slenderness ratio of $\sim 17$, which permits relative densities as low as $\sim 0.2{ }^{6}$ On the other hand, the decrease in $K_{c}{ }^{2} / \pi \sigma_{y}{ }^{2}$ with increasing $\sigma_{y} / \rho$, observed in essentially all classes of materials, ${ }^{8}$ is more limiting. For example, Vitreloy-1 struts must be thinner than $0.3 \mathrm{~mm}$ to effectively avoid fracture; such requirement severely limits fabricability.

MGMCs with optimal microstructures are found to exhibit both high strengths and ultra-high toughnesses (3). For example, when microstructurally optimized, MGMC (DH1) exhibits a strength of $\sim 1500 \mathrm{MPa}$ and a fracture toughness of $150 \mathrm{MPa} \mathrm{m}{ }^{1 / 2}$ (3 and 9). Owing to a large $\sigma_{y}$ and a relatively low $\rho, \sigma_{y} / \rho$ of the composite is comparable to that of Vitreloy-1 and substantially higher than the crystalline alloys. The elastic strain limit is not substantially different than Vitreloy-1, and hence the buckling resistance of the composite is likewise limited. Due to a very high $K_{I C}$ however, the fracture resistance of the composite is substantially higher than both Vitreloy-1 and Ti-6Al-4V and comparable to $\mathrm{Al}$ 6061 T6. The combination of such high $K_{c}^{2} / \pi \sigma_{y}{ }^{2}$ and $\sigma_{y} / \rho$ renders DH1 attractive for developing strong cellular structures with high mechanical energy absorption capacity.

Aside from superb mechanical properties, MGMCs possess a unique processing capability. Processability is considered key for achieving complex buckling-resistant periodic architectures suitable for mechanical energy absorption. Typical fabrication routes with traditional crystalline metals involve liquid-state as well as solid-state processes. ${ }^{10}$ Melt forming processes such as investment casting are suitable for the fabrication of complex architectures such as trusses, but are relatively expensive and limited to low-meltingtemperature high-fluidity alloys such as aluminum alloys. Moreover, the high fluidity of these melts gives rise to casting flaws and imperfections that invariably affect their mechanical performance. Solid-state forming processes are typically less costly, but are generally limited to rather simple architectures, especially when less ductile metal alloys are utilized (e.g., Ti alloys). In the temperature region between solidus and liquidus, MGMCs exhibit a very viscous semi- solid state consisting of a high-viscosity glass-forming eutectic liquid containing homogeneously distributed microscopic dendrites. The low fluidity of the semisolid state enables the use of forging processes to fabricate MGMC hardware. ${ }^{11}$

In this study, egg-box architectures were fabricated from DH1 via semisolid forging. The egg-box architecture is an attractive topology from a structural perspective. Deformation in the egg-box lattice is often accommodated by a traveling knuckle, and thus possesses much higher strain capacity than conventional honeycomb lattices that typically deform by rotation of a stationary hinge. ${ }^{12}$ To date, metallic egg-box panels for mechanical energy absorption have only been fabricated from commercially pure aluminum (Al 1050 H111) via cold superplastic press forming. ${ }^{12}$ The semisolidforging setup employed here is described in detail in Ref. 11. A split brass die is utilized consisting of positive and negative die tools with a checkerboard array of "up" and "down" pyramids. Water-cooling lines were soldered onto the back of each die tool to remove heat during processing. The lower die tool is shown inside the semisolid-forging setup in Figs. 1(a) and 1(b). The initial ingot and the as-forged egg-box panel are also shown. Pre-alloyed DH1 ingots $(\sim 25 \mathrm{~g})$ were heated inductively under an inert atmosphere to $\sim 950{ }^{\circ} \mathrm{C}$, i.e., above the solidus $\left(\sim 800^{\circ} \mathrm{C}\right)$ and substantially below the liquidus temperature $\left(\sim 1300{ }^{\circ} \mathrm{C}\right)$, and subsequently squeezed between the top and bottom die tools using $\sim 20 \mathrm{MPa}$ pressure. A forged panel with a relative density of 0.12 is shown in Fig. 1(c). The panel is of very uniform thickness (within $\pm 0.05 \mathrm{~mm}$ ), free of flaws and imperfections, and closely mimics the topology of a conventional egg-box carton [Figs. 1(c) and 1(d)]. The pyramidal cell width and height are 5 and $4 \mathrm{~mm}$, respectively, while the thickness is $0.5 \mathrm{~mm}$ [Fig. 1(e)]. The thickness is well below the plastic zone size of $\sim 3.1 \mathrm{~mm}$ (Table I), thereby ensuring adequate resistance to fracture. Moreover, the edge aspect ratio (height over thickness) of $\sim 10$ is well below the critical aspect ratio for buckling associated with the present architecture and material. Scanning electron microscopy performed across a section of a cell [Fig. 1(f)] reveals that the microstructure evolved sufficiently to enable high strength and toughness.

Egg-boxes panels with thicknesses ranging from 0.35 to $0.55 \mathrm{~mm}$ and relative densities ranging from 0.09 to 0.12 were quasistatically compressed using a screw-driven Instron. Strain was recorded using a linear variable differential transformer. The loading response of a 0.09 relative density panel is presented in Fig. 2(a). After a brief linear elastic response, the panel is seen to yield and collapse noncatastrophically at $\sim 30 \mathrm{MPa}$ stress. Following the first event, the panel continues to deform by successive reloading 

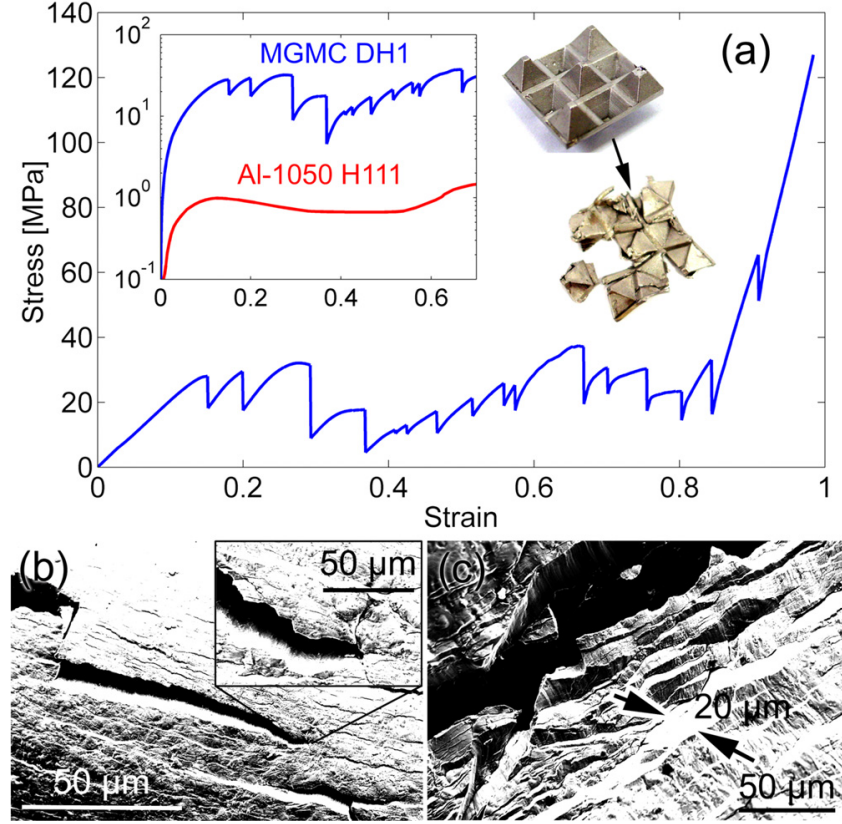

FIG. 2. (Color online) (a) Quasi-static compressive loading response of a DH1 egg-box panel of 0.09 relative density. Inset: logarithm of the stress strain response of 0.09 relative density DH1 and Al 1050 H111 egg-box panels along with images of the DH1 panel before and after collapse. (b) and (c) Scanning electron micrographs of a collapsed DH1.

and collapsing events while maintaining an average plateau stress of $\sim 20 \mathrm{MPa}$ up to a strain of $\sim 0.9$, at which point the panel becomes almost flat (with some segments being fragmented). The initial and fully collapsed states of the panel are presented as insets in Fig. 2(a). The mechanisms accommodating collapse are examined by scanning electron microscopy performed on fully collapsed cell segments, as shown in Figs. 2(b) and 2(c). In the micrograph in Fig. 2(b) deflection and plastic arrest of a propagated crack are revealed, consequences of the plastic interactions between the glassy matrix and ductile inclusion. The large cumulative plasticity enabled in the glassy matrix by such interactions is supported by evidence of long shear-sliding offsets $(\sim 20 \mu \mathrm{m})$, shown in Fig. 2(c). Hence, even though limited buckling and fracture appear to have occurred, the collapse process appears to have been accommodated predominantly by plastic yielding in the glassy matrix. Consequently, the failure and average plateau stress of the collapsing panels are unusually high. To put this stress level into prospective, the stress-strain response of the MGMC panel is plotted in the inset of Fig. 2(a) against that of an Al-1050 H111 panel of same relative density. As seen in the plot, the failure and average plateau stress of the MGMC panel far exceeds that of aluminum by a factor of $\sim 30$.

The specific mechanical energy absorbed by several collapsing panels (in $\mathrm{J} / \mathrm{g}$ ) was estimated up to the densification strain (i.e., where stress departs monotonically from the average plateau stress). The data are plotted in Fig. 3 against the corresponding relative density, together with data for various architectures made of conventional metallic alloys. As seen in the plot, the MGMC egg-box panels far outperform egg-box panels made of commercially pure aluminum ${ }^{12}$

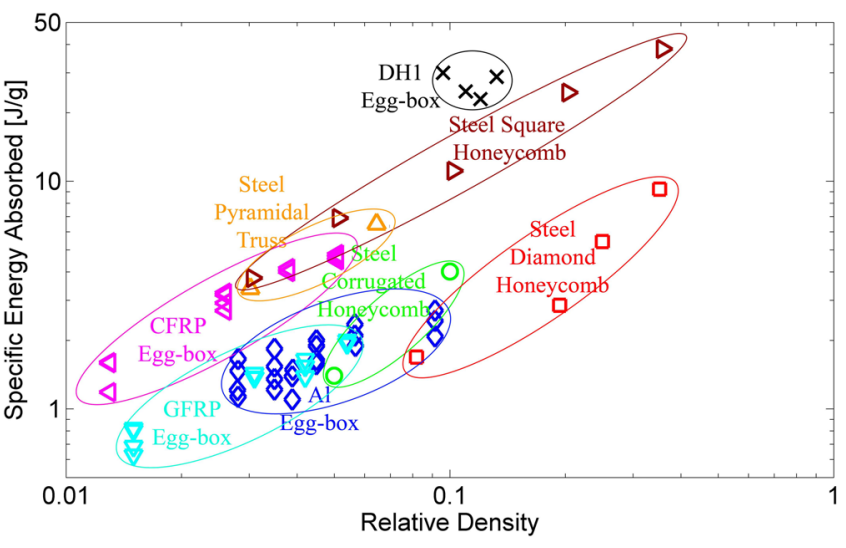

FIG. 3. (Color online) Estimated specific mechanical energy absorbed (in $\mathrm{J} / \mathrm{g}$ ) by $\mathrm{DH} 1$ panels plotted against the corresponding relative density. Data for various other architectures made of conventional metallic alloys and other engineering materials are also plotted.

or ultralight composites. More impressively, the present eggbox panels perform better than structurally advanced steel architectures such as pyramidal trusses and out-of-plane loaded square honeycombs, known to be much more resistant to elastic buckling than the egg-box architecture. ${ }^{13-15}$ The ability of the MGMC egg-box panels to outperform panels with architectures inherently much stronger is attributed to the unusually high strength and toughness of the MGMC enabling extensive plastic collapse at very high plateau stresses. Therefore, in terms of structural performance and ease of fabrication (and not counting other factors such as material cost etc.), one may conclude that MGMCs are attractive materials for cellular structure fabrication.

D.C.H. acknowledges support from the Jet Propulsion Laboratory, California Institute of Technology, under a contract with the National Aeronautics and Space Administration (NASA). The work was supported by the Office of Naval Research under Award No. N00014-07-1-1115.

${ }^{1}$ H. N. G. Wadley, Proc. R. Soc. London, Ser. A 364, 31 (2006).

${ }^{2}$ M. F. Ashby, Materials Selection in Mechanical Design (Pergamon, Oxford, 1992).

${ }^{3}$ D. C. Hofmann, J. Y. Suh, A. Wiest, G. Duan, M. L. Lind, M. D. Demetriou, and W. L. Johnson, Nature (London) 451, 1085 (2008).

${ }^{4}$ M. D. Demetriou, C. Veazey, J. S. Harmon, J. P. Schramm, and W. L. Johnson, Phys. Rev. Lett. 101, 145702 (2008).

${ }^{5}$ H. N. G. Wadley, N. A. Fleck, and A. G. Evans, Compos. Sci. Technol. 63, 2331 (2003).

${ }^{6}$ L. J. Gibson and M. F. Ashby, Cellular Solids: Structure and Properties, 2nd ed. (Cambridge University Press, Cambridge, 1997).

${ }^{7}$ See http://www. matweb.com for material property data by Automation Creations, Inc.

${ }^{8}$ M. E. Launey and R. O. Ritchie, Adv. Mater. 21, 2103 (2009).

${ }^{9}$ M. E. Launey, D. C. Hofmann, J. Y. Suh, H. Kozachkov, W. L. Johnson, and R. O. Ritchie, Appl. Phys. Lett. 94, 241910 (2009).

${ }^{10}$ H. N. G. Wadley, Adv. Eng. Mater. 4, 726 (2002).

${ }^{11}$ D. C. Hofmann, H. Kozachkov, H. E. Khalifa, J. P. Schramm, M. D Demetriou, K. S. Vecchio, and W. L. Johnson, JOM 61, 11 (2009).

${ }^{12}$ M. Zupan, C. Chen, and N. A. Fleck, Int. J. Mech. Sci. 45, 851 (2003).

${ }^{13}$ F. W. Zok, S. A. Waltner, Z. Wei, H. J. Rathbun, R. M. McMeeking, and A. G. Evans, Int. J. Solids Struct. 41, 6249 (2004).

${ }^{14}$ F. Côté, V. S. Deshpande, N. A. Fleck, and A. G. Evans, Mater. Sci. Eng., A 380, 272 (2004).

${ }^{15}$ F. Côté, V. S. Deshpande, N. A. Fleck, and A. G. Evans, Int. J. Solids Struct. 43, 6220 (2006) 